\title{
Conditions for formation of a kinetic anode layer in crossed $E \times B$ fields
}

\author{
A. Yu. Kovalenko and Yu. A. Kovalenko \\ State Unitary Enterprise “All-Russian Electrotechnical Institute," Moscow 111250, Russia \\ M. Keidar ${ }^{\text {b) }}$ \\ Department of Aerospace Engineering, University of Michigan, Ann Arbor, Michigan 48109
}

(Received 19 February 2006; accepted 10 June 2006; published online 4 August 2006)

\begin{abstract}
A discharge in crossed electric and magnetic fields $(E \times B)$ is considered. $E \times B$ discharge serves as a basis for Hall-effect plasma accelerators. In this letter the authors present conditions for formation of a kinetic anode layer by considering an interface between kinetic and hydrodynamic regions. It is found that the kinetic anode layer, which has a thickness of about the electron Larmor radius, is formed in the case of a high magnetic field. (C) 2006 American Institute of Physics.
\end{abstract}

[DOI: $10.1063 / 1.2240479]$

Formation of a region with strong electric field in a magnetized plasma remains an important problem in a number of areas such as magnetically insulated diode, ${ }^{1}$ transmission lines, ${ }^{2}$ magnetrons, ${ }^{3}$ plasma immersed ion implantation, ${ }^{4}$ and plasma thrusters. ${ }^{5}$ Despite having different applications these technologies are based on the same physical effect of using magnetic field in order to maintain a large electric field. One important consequence of the $E \times B$ field configuration is the formation of a large electron drift current. The $E \times B$ region serves as a basic for so-called Hall-effect plasma thrusters. ${ }^{5}$ Nowadays Hall thrusters, in general, are among the most advanced and efficient types of electrostatic propulsion devices. The electric field in the Hall thruster is perpendicular to the magnetic field and thus passing the electron current across a magnetic field leads to an electron closed drift or Hall drift, that provides the necessary gas ionization. In the Hall thruster the particle acceleration takes place in a quasineutral plasma and thus is not limited by space charge effects unlike the ion thruster counterpart. Two variants of Hall thruster exist: a thruster with closed electron drift in the dielectric channel and extended acceleration zone, or stationary plasma thruster, and a thruster with short acceleration region inside the metallic channel or thruster with anode layer (TAL). ${ }^{5}$ In a TAL, the ion acceleration takes place over a very short length of about the electron Larmor radius near the anode. This is why the term "anode layer" was attributed to the Hall thruster with metal walls.

The theory of the quasineutral $E \times B$ layer dubbed anode layer (AL) was developed over a few decades, however, a complete solution is not found yet. Probably the first detailed investigation of the $E \times B$ layer was performed by Chapman and Ferraro. ${ }^{6}$ They analyzed a problem of plasma interaction in a magnetic field in which both ions and electrons enter the boundary layer with the same velocity perpendicular to the magnetic field. It should be noted that this situation is somewhat different from Hall thrusters in which ion and electron fluxes are in opposite directions.

Hydrodynamic approaches for $E \times B$ region, which a priori assume quasineutrality, were developed. ${ }^{7-16}$ Such macroscopic approach relies on the assumption that the $E$ $\times B$ region extends beyond the electron Larmor radius. In this respect, one can argue that hydrodynamic models may

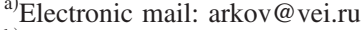

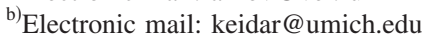

not be applied for AL, which in the case of uniform magnetic field has thickness of about the Larmor radius. ${ }^{7}$

Another approach to attack the problem of $\mathrm{AL}$ in $E \times B$ fields without assuming quasineutrality was recently presented. ${ }^{17}$ In Ref. 17 new classes of solutions were found, i.e., so-called $B$ and $E$ layers with distinct potential distributions as shown schematically in Fig. 1. It was shown ${ }^{17}$ that these solutions are different by the thickness of the ion acceleration region and by the parameters of the cathode plasma, which supplies electrons to AL. A quasikinetic approach was further developed in Ref. 18. This quasikinetic approach consists in coupled solution of charged and neutral particles motion taking into account ionization in the layer. Moreover, the integration of the derived equations is performed starting from the anode, which required modification of the system of equations. The main distinction of the quasikinetic approach from the hydrodynamic formulation consists in the description of the electron transport without introducing macroscopic conductivity (classical or anomalous). Transport of the electron component is determined by magnetic and electric fields in the AL. Electrons enter the AL from the cathode side, begin its cycloidal trajectory under $E \times B$ fields, and reach the anode boundary before returning to the cathode surface as shown schematically in Fig. 1. A self-magnetic field generated by the electron drift current and leading to demagnetization (described below) of the AL is included. Typically, a very large electron current is circulated in the AL with a very small fraction being scattered and reach the anode. ${ }^{18}$ Thus, most electrons (about 90\%) are col-

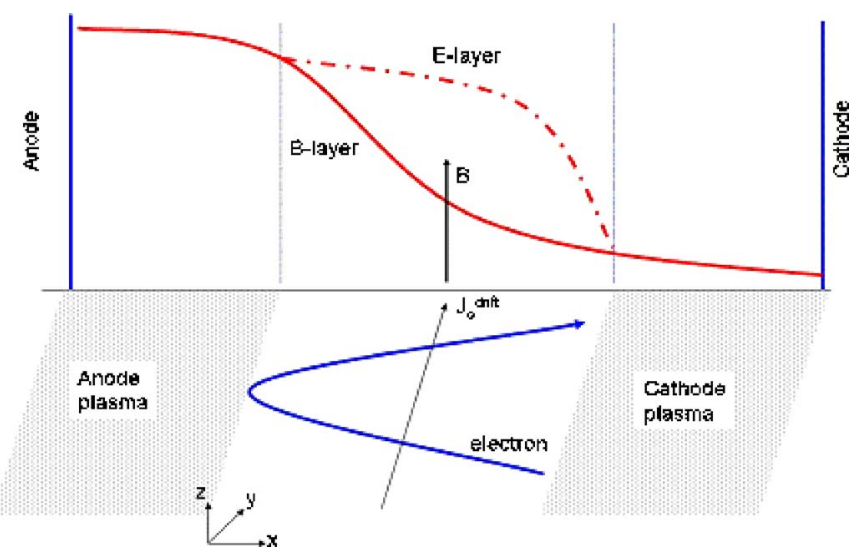

FIG. 1. (Color online) Schematic of the anode layer and potential distribution in the Hall thruster with anode layer. 
lisionless that justifies the kinetic approach. ${ }^{18}$ It is important to understand the accuracy and applicability of this approach. In this letter we consider applicability of this approach for analysis of the AL in the $E \times B$ fields. The reliable parameter envelope for application of the quasikinetic approach will be established by "lacing" a solution of the kinetic region with the hydrodynamic solution as will be explained below. In other words the main impetus of this letter is to determine conditions for formation of the kinetic $\mathrm{AL}$ in $E \times B$ discharge.

Briefly the mathematical model consists in solving the Poisson equation, Ampere law, and equation of motion for electrons and ions. Electron flow arising at the cathode reaches the anode and then returns back to the cathode moving in the opposite direction as a result of the magnetic field effect. Therefore, two electron flows are present in the AL. On the other hand the cathode plasma is assumed to be collision dominated. ${ }^{8}$ In order to determine the AL thickness, it is necessary to find conditions at the interface between the kinetic (anode layer) and hydrodynamic (cathode plasma) regions as shown in Fig. 1. It is considered that at the interface between these two regions an electrical field, a plasma density and its gradient, a potential, and a magnetic field are continuous functions. Under the above assumptions, the problem can be described by the following set of equations:

$$
\begin{aligned}
& \frac{d V_{x}^{e}}{d x}=E_{x}+V_{y}^{e} \times B, \\
& \frac{d V_{y}^{e}}{d x}=-V_{x}^{e} \times B, \\
& \frac{d B}{d x}=\frac{-4 \pi e}{c} j, \\
& \frac{d^{2} \varphi}{d x^{2}}=4 \pi e\left(n_{e}-n_{i}\right),
\end{aligned}
$$

where $V^{e}$ is the electron velocity, $j$ is the current density in $\mathrm{AL}, B$ is the magnetic field in AL with demagnetization factor (the self-magnetic field which is generated in the layer decreases the externally applied magnetic field), and $n_{i}$ $=\sum_{m=1}^{k} j_{m}^{+}(x) /\left|V_{m}^{+}(x)\right|$ is the ion density, which is found via current tube method as described in detail elsewhere. ${ }^{18}$

It should be noted that integration of Eqs. (1)-(4) is performed from the anode by assigning reverse electron flow. In this formulation of the AL problem we assumed that electron energy at the "cut off" point (i.e., at the anode) is close to the applied total potential drop. In other words, this means that the potential drop in the cathode plasma region is assumed to be negligible. Thus, the validity of this approach can be established by calculation of a potential drop in the AL and comparison of this potential drop with the total discharge voltage.

Recall that similarly to Ref. 18 we employ in this steady state formulation the substitution of variables in the form $V_{x}^{e}=d x / d \tau$ for convenience, which helps us to overcome the mathematical problem of electron density singularity at the anode side of AL.

We use the continuity conditions for the electron flux at the cathode boundary, i.e., $j_{e x}=j_{e x}^{h}$ and $j_{e y}=j_{e y}^{h}$, in order to

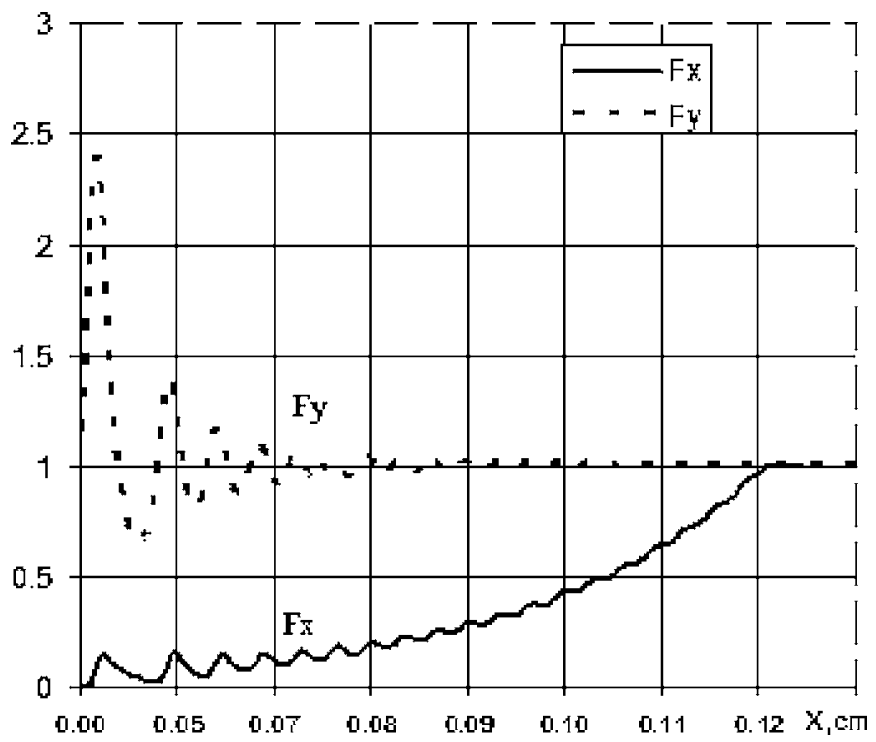

FIG. 2. Spatial distribution of functions $F y$ and $F x$ in the kinetic layer. Discharge voltage $U_{d}=200 \mathrm{~V}$, mass flow rate $q=1.36 \mathrm{mg} / \mathrm{s}$, and magnetic field $H=200 \mathrm{G}$ (discharge current calculated by the model is $I_{d}=1.66 \mathrm{~A}$ ).

determine the interface between the kinetic and hydrodynamic regions. One can obtain electron fluxes in the hydrodynamic region:

$$
\begin{aligned}
& j_{e x}^{h}=b_{\perp} \cdot n_{e} \cdot \frac{d \varphi}{d x}, \\
& j_{e y}^{h}=n_{e} \cdot \frac{c}{B} \cdot \frac{d \varphi}{d x} .
\end{aligned}
$$

By calculating electron current densities in the kinetic and hydrodynamic regions we determine the interface as a position at which electron fluxes are equal. The region beyond the interface, between the interface and cathode, is considered to be collision dominated. Consequently, this approach allows determination of the thickness of the kinetic region.

As an example we show the calculated dependence of the functions $\mathrm{Fy}=j_{e y} / j_{e y}^{h}$ and $\mathrm{Fx}=j_{e x} / j_{e x}^{h}$ as a function of the axial position along the kinetic layer. The calculations show (see Fig. 2) that Fy varies only weakly along the AL and thus the AL cathode boundary is determined by the condition $F x=1$. It was found that both functions oscillate in space as shown in Fig. 2. The oscillatory character of these functions near the anode boundary is caused by the space charge oscillations due to ion production and acceleration in the AL, as was previously reported. ${ }^{18}$ However, the nature of these oscillations and their dependence on the external parameters require further investigation and goes beyond the scope of this letter. It should be also pointed out that similar oscillations were observed in the case of small ion velocity near the sheath edge. ${ }^{19}$

By defining the interface between the kinetic and hydrodynamic regions one can calculate the fraction of the voltage in the kinetic region. A relative fraction of the potential drop in the kinetic region is shown in Fig. 3. One can see that this fraction increases with magnetic field. In the low magnetic field range $(50-100 \mathrm{G})$ the voltage drop across the kinetic region is relatively small, thus AL in this case is essentially hydrodynamic. Recall that in this range of parameters, the present approach for modeling the kinetic region is not ac- 


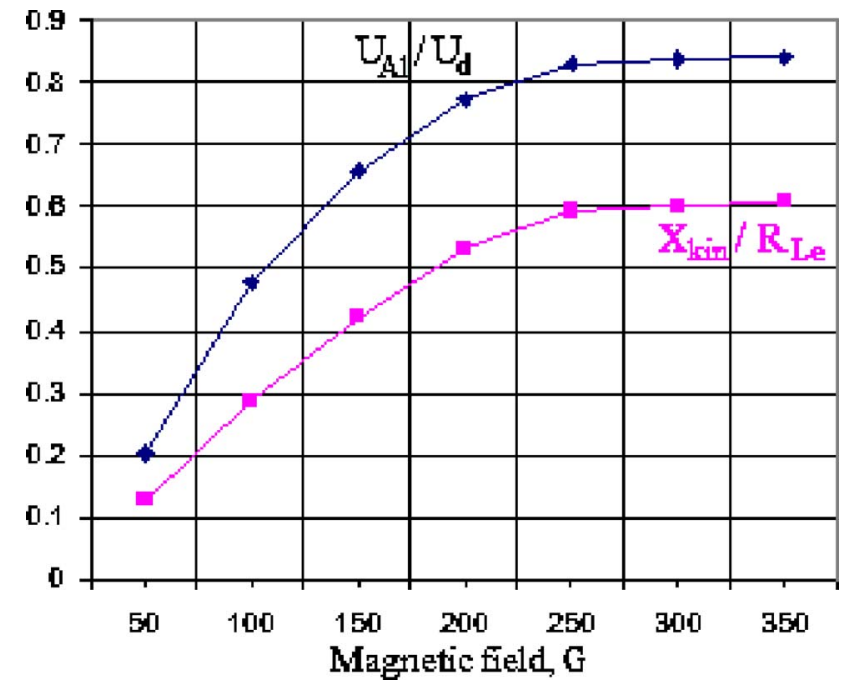

FIG. 3. (Color online) Dependences of the relative potential drop in the kinetic region $\left(U_{\mathrm{AL}} / U_{d}\right)$ and the normalized thickness of the kinetic region $\left(X_{\text {kin }} / R_{L e}\right)$ on the magnetic field. $U_{d}=200 \mathrm{~V}$ and $q=1.36 \mathrm{mg} / \mathrm{s}$.

curate, since it relies on the assumption that the main fraction of the discharge voltage falls in the kinetic region.

In this model the classical conductivity was used for electron velocity derivation in the hydrodynamic region. This assumption leads to saturation of the normalized thickness of the kinetic region with magnetic field as shown in Fig. 3. It can be seen that the thickness of the kinetic region is the about the electron Larmor radius, in agreement with previous models. 6,7

Results shown in Fig. 3 suggest that higher magnetic field leads to a smaller fraction of the voltage in the hydrodynamic region. Thus AL becomes essentially a kinetic region with a thickness of about the electron Larmor radius. This result is related to the broader question about the maintenance of an electric field in the partially magnetized plasma, i.e., whether potential drop is concentrated in the narrow region of about the electron Larmor radius as was suggested earlier by Zharinov ${ }^{7}$ or it is extended over a larger region of about the mean free path i.e., hydrodynamic region (Refs. 8 and 9). Assuming that a balance is established between electron production and electron losses due to crossfield transport one can arrive to the following estimation of the AL thickness: ${ }^{20}$

$$
L=R_{L e} \sqrt{\frac{\nu_{\text {eff }}}{\nu_{\text {ion }}}}
$$

where $R_{L e}$ is the electron Larmor radius, $\nu_{\text {eff }}$ is the effective collision frequency, and $\nu_{\text {ion }}$ is the ionization frequency. It can be seen from Eq. (7) that if the effective collision frequency is much larger than the ionization frequency the anode layer extends beyond the Larmor radius. On the other hand smaller effective collision frequency to ionization frequency ratio leads to thin $\mathrm{AL}$ with thickness of about the Larmor radius. In general, magnetic field reduces effective collision frequency, thus leading to thin AL. This conclusion is consistent with our results (see Fig. 3) that show that higher magnetic field causes formation of the kinetic AL with smaller voltage fraction in the hydrodynamic region.

Figure 4 shows the region of validity of the quasikinetic approximation for description of the AL. In this particular

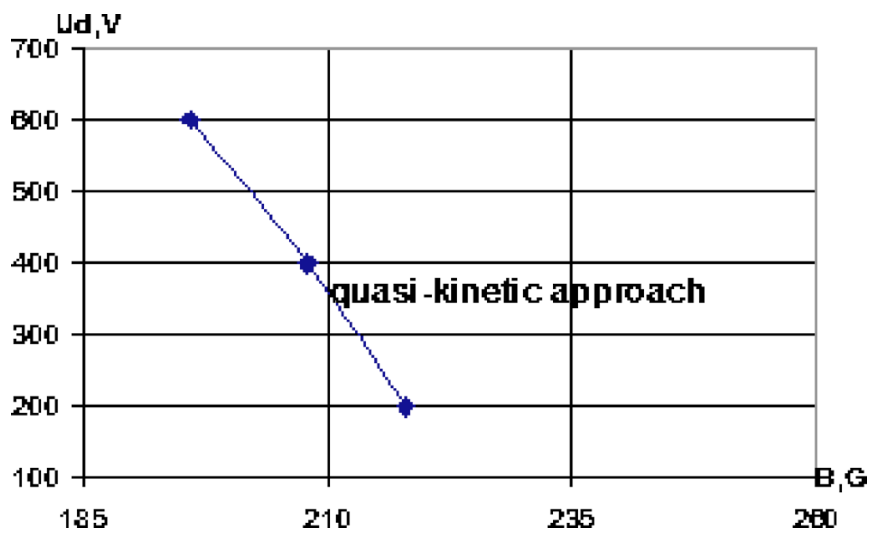

FIG. 4. (Color online) Region of validity for the quasikinetic approximation (based on the condition that less than $20 \%$ of the discharge voltage falls in the hydrodynamic region). Xenon flow rate is $1.36 \mathrm{mg} / \mathrm{s}$.

example a condition that less than $20 \%$ of the discharge voltage falls in the hydrodynamic region was employed. In the case of a higher magnetic field the main fraction of the voltage falls across the length of about the Larmor radius, i.e., across the kinetic anode layer. Again, it should be pointed out that this result is in agreement with simple AL analysis [Eq. (7)] described above.

In conclusion a one-dimensional kinetic model of a discharge in crossed electric and magnetic fields, which serves as a basis for Hall-effect plasma accelerators, was developed. The validity of this approach was demonstrated by considering the interface between the kinetic and hydrodynamic regions. It was shown that quasikinetic approach could be justified in the case of high magnetic field and high discharge voltage.

The authors thank Professor A.V. Zharinov for very fruitful discussions. This work was supported by Russian Federal Agency on Science and Innovation Grant No. 02.444.11.7031 and RFBR Grant No. 05-02-08030.

${ }^{1}$ I. I. Beilis, A. Fruchtman, and Y. Maron, IEEE Trans. Plasma Sci. 26, 995 (1998).

${ }^{2}$ K. D. Bergeron, Appl. Phys. Lett. 28, 306 (1976).

${ }^{3}$ S. M. Rossnagel and H. R. Kaufman, J. Vac. Sci. Technol. A 5, 88 (1987).

${ }^{4}$ M. Keidar, O. R. Monteiro, A. Anders, and I. D. Boyd, Appl. Phys. Lett. 81, 1183 (2002).

${ }^{5}$ V. V. Zhurin, H. R. Kaufman, and R. S. Robinson, Plasma Sources Sci. Technol. 8, 1 (1999).

${ }^{6}$ S. Chapman and V. Ferraro, J. Geophys. Res. 57, 15 (1952).

${ }^{7}$ A. V. Zharinov and Yu. S. Popov, Sov. Phys. Tech. Phys. 12, 208 (1967).

${ }^{8}$ V. K. Kalashnikov and Yu. V. Sanochkin, Sov. J. Plasma Phys. 7, 303 (1981).

${ }^{9}$ A. I. Morozov and V. V. Savel'ev, Plasma Phys. Rep. 26, 219 (2000).

${ }^{10}$ M. Keidar, I. D. Boyd, and I. I. Beilis, Phys. Plasmas 8, 5315 (2001).

${ }^{11}$ E. Ahedo, Phys. Plasmas 8, 3058 (2001).

${ }^{12}$ A. Fruchtman, N. J. Fisch, and Y. Raitses, Phys. Plasmas 8, 1048 (2001).

${ }^{13}$ S. Barral, K. Makowski, Z. Peradzyski, N. Gascon, and M. Dudeck, Phys. Plasmas 10, 4137 (2003).

${ }^{14}$ M. Keidar, I. D. Boyd, and I. I. Beilis, Phys. Plasmas 11, 1715 (2004).

${ }^{15}$ Y. Raitses, M. Keidar, D. Staack, and N. J. Fisch, J. Appl. Phys. 92, 4906 (2002).

${ }^{16}$ L. Dorf, V. Semenov, and Y. Raitses, Appl. Phys. Lett. 83, 2551 (2003).

${ }^{17}$ M. A. Vlasov, A. V. Zharinov, and Yu. A. Kovalenko, Tech. Phys. 46, $1522(2001)$.

${ }^{18}$ A. Yu. Kovalenko and Yu. A. Kovalenko, Tech. Phys. 48, 1413 (2003).

${ }^{19}$ M. Keidar and I. I. Beilis, IEEE Trans. Plasma Sci. 33, 1481 (2005).

${ }^{20}$ V. E. Erofeev and L. V. Leskov, in Physics and Application of Plasma Accelerators, edited by A. I. Morozov (Nauka and Technika, Minsk, 1974), p. 18 (in Russian). 